\title{
Optimal Operation of Combined Heat and Power Systems: An Optimization-Based Control Strategy
}

\author{
Jenny L. Diaz C.,*, Carlos Ocampo-Martinez ${ }^{\mathrm{a}}$, Niklas Panten ${ }^{\mathrm{b}}$, Thomas Weber ${ }^{\mathrm{b}}$, Eberhard Abele ${ }^{\mathrm{b}}$ \\ ${ }^{a}$ Automatic Control Department, Universitat Politècnica de Catalunya, Institut de Robòtica i Informàtica Industrial (CSIC-UPC), Llorens i Artigas 4-6, Planta 2, \\ 08028 Barcelona, Spain \\ ${ }^{b}$ Institute of Production Management, Technology and Machine Tools (PTW), Otto-Berndt-Str. 2, 64287 Darmstadt, Germany
}

\begin{abstract}
The use of decentralized Combined Heat and Power (CHP) plants is increasing since the high levels of efficiency they can achieve. Thus, to determine the optimal operation of these systems in dynamic energy-market scenarios, operational constraints and the time-varying price profiles for both electricity and the required resources should be taken into account. In order to maximize the profit during the operation of the CHP plant, this paper proposes an optimization-based controller designed according to the Economic Model Predictive Control (EMPC) approach, which uses a non-constant time step along the prediction horizon to get a shorter step size at the beginning of that horizon while a lower resolution for the far instants. Besides, a softening of related constraints to meet the market requirements related to the sale of electric power to the grid point is proposed. Simulation results show that the computational burden to solve optimization problems in real time is reduced while minimizing operational costs and satisfying the market constraints. The proposed controller is developed based on a real CHP plant installed at the ETA research factory in Darmstadt, Germany.
\end{abstract}

Keywords: Combined heat and power systems, Profit maximization, Economic model predictive control, Mixed Integer Linear Programming, Non-constant time-step size

\section{Introduction}

The energy supply in Germany is increasingly changing from a centralized to a decentralized generation structure [1]. Besides the installation of renewable energies, the use of decentralized Combined Heat and Power (CHP) plants is increasing, as they can achieve high levels of fuel utilization [2]. However, the cost efficiency of a CHP is highly dependent on the operation strategy and, therefore, identifying an optimal operation strategy in volatile energy markets while covering thermal demands can be challenging though. To this end, optimization algorithms are often used for scheduling the activation instants of the CHP system, which can be determined according to the operating constraints of both the plant and the system itself [3].

In addition to operating constraints, the optimal operation of a CHP plant from an economic point of view is limited by the costs of the required resources (for instance, natural gas), the sale price of the electric power in the market, and its fluctuations over time. However, to take price fluctuations on the electricity market into account when choosing an operation strategy for CHPs, a sufficiently large optimization horizon is required. Particularly, for an intraday optimization, this horizon is usually assumed to be about one day, which is usually modeled by using a temporal resolution of 15 minutes that implies a high computational cost [4]. In [5], an optimization procedure is

\footnotetext{
${ }^{*}$ Corresponding author

Email address: jenny. Iorena.diaz@upc. edu (Jenny L. Diaz C. )
}

proposed based on non-linear optimization techniques and it is applied to the determination of day ahead of operation program with a 15-minutes time step. On the other hand, in order to maximize the revenue by selling the generated electric power or its excess, it must be ensured that the plant actually produces the amount of electricity traded with the electric company to avoid economic penalties. From this fact, a higher temporal resolution to increase the degrees of freedom during the CHP operation, and more accurate modeling to achieve a proper representation of CHP behavior could be required [6]. Therefore, if both a long time horizon and a high temporal resolution are chosen, the number of decision variables taken into account in the optimization problem increases rapidly. This last issue is aggravated by an increased requirement for model accuracy when mapping the transient behavior of the plant. Thus, in order to solve the optimization problem within a short time, the number of considered decision variables must be kept low but in a way that allows the required accuracy.

According to the reported literature [7], the strategies proposed so far for planning and scheduling of CHP systems are focused on achieving a low operation cost, minimization of resources, or minimization of waste. However, most of these strategies are designed considering energy prices and the thermal and electric power demand to be constant for a long period of time. Thus, as a consequence, the CHP system is not able to be optimized in real-time since the current conditions of both the system and energy market will not be updated. In [8], one way to reduce the complexity of the problem is proposed by 


\section{Nomenclature}

\section{Physics Constants}

CHP Combined Heat and Power

CVA Canonical Variate Analysis

EMPC Economic Model Predictive Control

$\mathrm{EMPC}_{1} \mathrm{EMPC}$ controller with non-constant time step

$\mathrm{EMPC}_{2}$ EMPC controller with constant time step

LTI Linear time-invariant

MOESP Multivariable Output Error State Space

MPC Model Predictive Control

N4SID Numerical algorithm For Subspace Identification

RBC Rule-based control

SI Subspace identification

TES Thermal energy storage

\section{Number Sets}

$\begin{array}{ll}\mathbb{R} & \text { Real Numbers } \\ \mathbb{Z} & \text { Integer Numbers } \\ \mathbb{Z}_{\geq 0} & \text { Positive integer Numbers }\end{array}$

\section{Symbols}

$\Delta t \quad$ Sampling time

$\hat{x} \quad$ State estimation

$\mathbb{Z}_{\geq 0} \quad$ Positive integer Numbers

u Input vector

$\mathbf{v} \quad$ Measurement noise

w State noise

$\mathbf{x}$ State vector

y Output vector

$\bar{P}_{E} \quad$ Upper bound for electric power generation

$\bar{Q}_{T E S} \quad$ Maximum thermal power in the TES

$\bar{T}_{2} \quad$ Upper bound for output temperature in the CHP

$\theta_{1} \quad$ Cost by gas consumption

$\theta_{2} \quad$ Depreciation cost

$\theta_{3} \quad$ Switching cost

$\theta_{4} \quad$ Total revenues

$\theta_{5} \quad$ Difference between traded and produced electric power

$\underline{P}_{E} \quad$ Lower bound for electric power generation

$\underline{Q}_{T E S} \quad$ Minimum thermal power in the TES

$\underline{T}_{2}^{T E S} \quad$ Lower bound for output temperature in the CHP

$|\mathbf{x}| \quad$ Cardinality of vector $\mathbf{x}$
$A_{T E S} \quad$ Transfer area of TES

$b \quad$ Number of input signals

$C_{p} \quad$ Heat capacity

$F_{G} \quad$ Volumetric flow of gas

$F_{W} \quad$ Volumetric flow of water

$f_{s w} \quad$ Switching indicator for the CHP system

$H_{p} \quad$ Prediction horizon

$J \quad$ Total profit

$k \quad$ Discrete time index

$l_{e} \quad$ Economic cost function

$N \quad$ Model order

$O_{1} \quad$ Extended observability matrix

$p \quad$ Number of output signals

$P_{E} \quad$ Electric power

$P_{r, e} \quad$ Sale price of electric power

$P_{r, g} \quad$ Gas price

$P_{r, o n} \quad$ Price of keeping the system on

$P_{r, s} \quad$ Switching price

$P_{\text {tra }} \quad$ Economic penalty in the traded power

$P_{\text {tra }} \quad$ Traded electric power

$q_{C H P} \quad$ Thermal power generated by CHP

$q_{\text {dem }} \quad$ Thermal power demand

$q_{\text {loss }} \quad$ Energy losses with the environment

$Q_{T E S} \quad$ Thermal energy stored in TES

$T \quad$ Operation time

$t \quad$ Continuous time

$T_{1} \quad$ Temperature of water flow at the CHP inlet

$T_{2} \quad$ Temperature of water flow at the CHP output

$t_{c} \quad$ Computational time

$T_{s} \quad$ Total simulation time

$t_{s} \quad$ Controller time step

$T_{\text {env }} \quad$ Outside temperature

$T_{T E S} \quad$ Temperature in the TES

$T_{u d} \quad$ Updating period for traded electric power

$U \quad$ Global coefficient of heat transfer

$u \quad$ System input

$x \quad$ Model state

$\mathbf{V}^{*} \quad$ Optimal input sequence to CHP system

V Input sequence to CHP system dividing it into a planning problem and a fulfillment or adaptation (tracking) problem. The longer-term energy marketing is considered in the planning problem and then given as a target to the tracking problem. In addition, a two-stage stochastic programming model for CCHP-microgrid operation considering demand uncertainty is proposed in [9]. However, with the introduction of the continuous intraday market, the boundaries between the planning and tracking phases might become blurred. Therefore, to make the best possible use of the resulting optimization opportunities on the market, an approach that combines the optimization of both problems in a single model is required.

In this regard, optimization-based control strategies have gained attention since they allow considering the energy-price fluctuations and the system dynamics as constraints into an optimization problem behind the controller design. Among these strategies, the Model Predictive Control (MPC) approach has had a great application in the tracking stages once the planning stages have been previously optimized [10]. In [11], a stochastic MPC framework to optimally schedule and control the CHP microgrid with large-scale renewable energy sources to reduce the negative impacts introduced by uncertainties is proposed. 
Nevertheless, due to the need to combine both planning and tracking problems as well as the increasing interest of improving the economic performance of the CHP systems, the Economic Model Predictive Control (EMPC) has gained attention during the last years with great applications in systems such as the boiler-turbine system[12], residential building energy systems [13], and mechanical pulping processes [14]. The main advantage of EMPC with respect to MPC is that the former directly optimizes an economic cost function of the process, from which both market constraints and time-varying price profiles could be considered into both the cost function and the constraints of an optimization problem $[15,16]$. Thus, using the EMPC approach, both the planning and fulfillment problems could be addressed in one stage. Besides, according to the receding horizon principle [17] for the real-time implementation of control strategies, every time that the optimization problem is solved, the current cost/price information could be updated.

On the other hand, regarding model accuracy several approaches have been developed in the literature for the CHP plants, which are mainly focused on phenomenological-based models [18]. For instance, in [19], a modeling approach for a CHP plant that considers the effect of power gradients for depicts dynamic power changes more accurately than existing approaches is presented, while in [20], a multi-objective optimization model based on the technical, economic and environmental performances is developed. However, it is well known that phenomenological-based models require a high computational load because of the complexity of mathematical expressions used for representing the main phenomena that govern the system behavior. In addition, in most of the cases, model parameters, constants, or variables cannot be measured, estimated or determined in real environments. Consequently, datadriven models have become a useful alternative to model complex and large-scale systems [21]. Among the methods for getting models based on real data sets, the Subspace Identification (SI) algorithms were increasingly applied for the design of control strategies during the last years. The main reason for their widespread use is due to the fact that these methods directly deliver a linear state-space realization, which is quite useful in the design of control strategies [22]. In Table 1 some of the most relevant works during the last years are presented and classified according to the topics addressed for determining the optimal operation of CHP systems. From this review, it is possible to see that there is not a control strategy that integrates all the relevant features in only one strategy, apart of having real-time implementation capabilities.

Therefore, in order to solve the issues with respect to long optimization horizons, temporal resolution, and model accuracy, the main contribution of this paper is the design of a predictivelike controller based on the EMPC approach and by using a non-constant time-step size along the prediction horizon. The time-step width along the prediction horizon increases for time steps that lie further in the future, since if only the most recent time step is actually executed, model accuracy can be maintained while the number of decision variables is reduced. In this regard, the general idea of the proposed controller is to predict both the thermal and electrical power production that maximize
Table 1: Main research topics of the control strategies implemented for CHP systems.

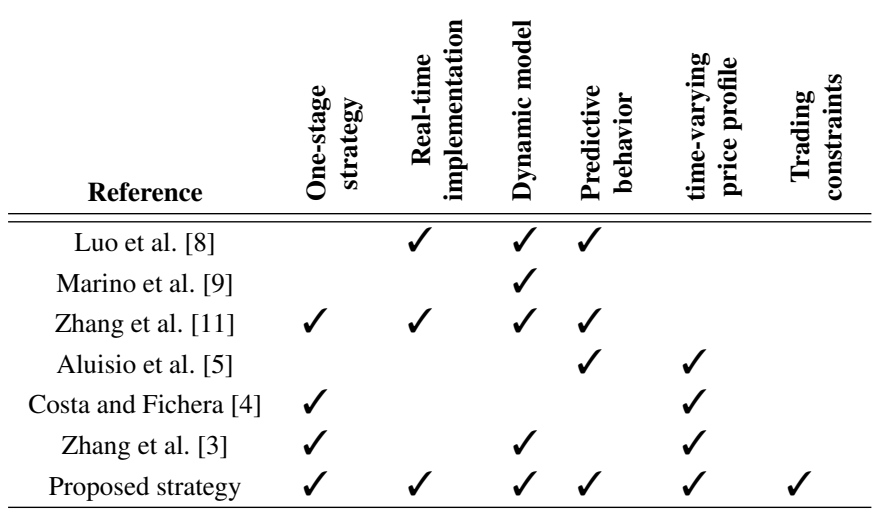

the profit during the CHP operation considering both operating and energy market constraints. Thus, the proposed controller will be designed based on a model for the operation of a CHP plant obtained by using SI methods and real data sets. In addition, in order to get the maximum profit regarding the sale of the electric power generated, a soft constraint for minimizing the difference between the generated and traded electric power is proposed, taking advantage of the high temporal resolution for the near future reached by using a non-constant time-step size.

It should be noted that in [23], a predictive-like controller was proposed using a non-constant time step as a first approximation to determine the optimal operation of cogeneration plants. However, in [23], energy losses due to the heat exchange with the environment and suitable measurements to avoid the high switching frequency of system actuators (e.g., valves, pumps) were not considered into the controller design. Thus, in contrast to the previous work, the proposed controller in this paper considers the energy losses in the process model, and in addition to energy market constraints and its fluctuations, safety constraints to avoid damages in the system are proposed in the controller design. Besides, the proposed controller is compared with another controller using a constant time step and more decision variables along the prediction horizon, in order to check the suboptimality of the proposed approach.

The remainder of the paper is organized as follows. In Section 2, the tools employed for the design of the proposed control strategy are briefly described. Then, the problem of maximizing profit for the operation of CHP plants is introduced in Section 3. Next, in Section 4, the proposed approach and the general idea about the non-constant time step are both presented and discussed. Then, a detailed description of the case study is presented in Section 5. Afterward, the obtained simulation results for the proposed approach are reported and analyzed in Section 6. Finally, conclusions and future work are drawn in Section 7. 


\section{Preliminaries}

\subsection{Economic Model Predictive Control}

Model predictive control (MPC) is an optimization-based control technique in which a cost function is optimized over a prediction horizon according to a dynamic process model and process constraints. According to [24], the main idea underlying MPC is to transform a control problem into an optimization one, in a way that at sampling time a sequence of future control values is computed. Usually, MPC is formulated using a quadratic objective function to penalize the deviations of the state and outputs of a system from their optimal steady-state values over a prediction horizon $[15,10]$.

Although the conventional MPC approach has had a great application, it does not allow a suitable representation of the economic performance of the processes. According to [16], there is an increasing number of problems for which dynamic economic performance is crucial and the hierarchical separation of economic analysis and control is either inefficient or inappropriate. Thus, in order to perform in a joint manner the process economic optimization and process control, a new MPC scheme has been proposed, in which the conventional tracking function is replaced by an economic cost function and, it is called Economic MPC (EMPC). In this regard, given that the EMPC directly optimizes the process economics, it has been widely used in the context of the manufacturing industry with the aim to determine the optimal operation of manufacturing systems from an economic viewpoint.

According to [15], the EMPC approach is characterized by the following optimization problem:

$$
\min _{u \in S} \int_{0}^{H_{p}} l_{e}(x(t), u(t)) d t
$$

subject to

$$
\begin{aligned}
\dot{x}(t) & =h(x(t), u(t), 0) \\
x(0) & =x\left(t_{k}\right) \\
g(x(t), u(t)) & \leq 0, \forall t \in\left[0, H_{p}\right)
\end{aligned}
$$

being $u$ the input trajectory of the decision variables over the prediction horizon $H_{p}, x$ the predicted state trajectory, $h(\cdot)$ the mathematical expressions for the nominal process model, $x(0)$ the initial conditions on the dynamic model, $g(\cdot)$ the process constraints, and $l_{e}(\cdot)$ is the process economic cost function that the EMPC optimizes through dynamic operation of the process.

It should be noted that the implementation strategy of the EMPC is the same as for the conventional MPC, i.e., in a receding horizon fashion. Several works related to the design of EMPC controllers, the theoretical background and stability analysis of EMPC have been proposed in the literature. A detailed explanation of the EMPC strategy can be found in $[16,25]$. On the other hand, some relevant applications of EMPC in industrial environments are presented in [26] for the building climate control in a Smart Grid, [12] boiler-turbine systems, and [14] for the mechanical pulping processes.

\subsection{Subspace identification}

Subspace identification (SI) methods allow identifying the matrices of a state-space realization of linear time-invariant (LTI) systems based on input-output data. These algorithms are useful since state-space realizations are convenient for estimation, control and prediction tasks. Many SI methods are based on algorithms that use both the observability and controllability matrices to determine the model matrices from input-output data for a real system.

Basically, SI methods start from the idea that a set of measurements of $b$ input signals $(b \geq 1)$ and $p$ output signals $(p \geq 1)$ satisfy an $N$-order state-space (unknown) realization as follow:

$$
\begin{aligned}
\mathbf{x}(k+1) & =A \mathbf{x}(k)+B \mathbf{u}(k)+\mathbf{w}(k), \\
\mathbf{y}(k) & =C \mathbf{x}(k)+D \mathbf{u}(k)+\mathbf{v}(k),
\end{aligned}
$$

where $k \in \mathbb{Z}_{\geq 0}$ corresponds to the discrete-time index, $\mathbf{x} \in$ $\mathbb{R}^{\ell}, \mathbf{u} \in \mathbb{R}^{b}$, and $\mathbf{y} \in \mathbb{R}^{p}$ are the state, input and output vectors, while $\mathbf{w} \in \mathbb{R}^{\ell}$ and $\mathbf{v} \in \mathbb{R}^{p}$ are the state noise and output measurement noise, respectively.

Thus, in a deterministic case, in which $\mathbf{w}$ and $\mathbf{v}$ are neglected, the SI problem consists of [27]

(a) Estimate the system order $N$.

(b) Estimate the system matrices $A \in \mathbb{R}^{\ell \times \ell}, B \in \mathbb{R}^{\ell \times b}, C \in$ $\mathbb{R}^{p \times \ell}$, and $D \in \mathbb{R}^{p \times b}$.

According to [27], two different families of SI algorithms have been identified and reported in the literature. The first family uses the state estimation $\hat{x}$ to determine the model matrices, while the second family uses the extended observability matrix $O_{i}$ to first determine estimates of matrices $A$ and $C$, and then, to estimate matrices $B$ and $D$. Some examples of the first family of algorithms are the Canonical Variate Analysis (CVA) and Numerical algorithm For Subspace IDentification (N4SID), while a representative example of the second family is the Multivariable Output Error State Space (MOESP) algorithm. The algorithms of the first family are based on the system theory, the unifying theorem, linear algebra, and they can be generalized in two main steps:

1. Determine $N$ and a state sequence $\hat{x}_{1}, \hat{x}_{2}, \cdots, \hat{x}_{n}, \hat{x}_{d+1}$. To this end, the data block Hankel matriz and the singular value decomposition are employed.

2. Solve a least-squares problem to obtain the state-space matrices based on state estimation $\hat{\mathbf{x}}$, and the measurements $\mathbf{u}$ and $\mathbf{y}$.

The last procedure is deeply explained in [28], while some of the previously mentioned SI algorithms, and the way they are implemented, are widely explained in [22, 27]. 


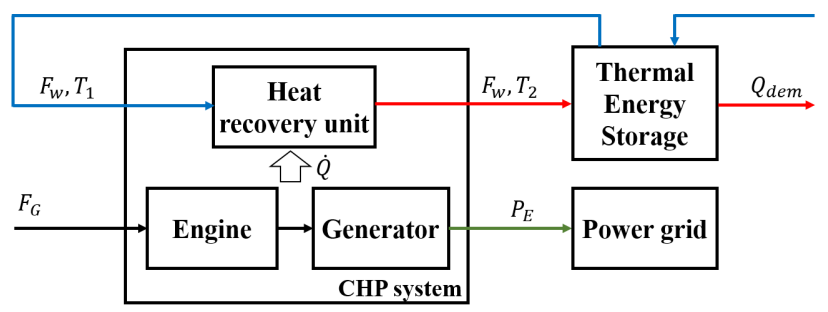

Figure 1: Combined Heat and Power (CHP) system with an integrated Thermal Energy Storage (TES) unit.

\section{Problem Statement}

The CHPs commonly refer to systems formed by a power generator and a heat recovery unit to produce electricity and useful heat at the same time. These systems are often based on gas engines to generate electric power and thermal energy for heating a water stream. In most cases, the heat recovered is used to directly cover the thermal power demand of buildings, other industrial processes, or it is transported towards a Thermal Energy Storage (TES) unit for its later use. A typical scheme of a CHP system with an integrated TES is shown in Figure 1.

According to Figure 1, the volumetric flow of gas $F_{G}$ is fed to the CHP system for generating electric power $P_{E}$, which is usually used by the processes in the plant or injected in the local grid point of common connection for its sale. During the combustion process of $F_{G}$, the heat generated as waste is recovered by heating a cooling fluid (e.g. water) from temperature $T_{1}$ to $T_{2}$ via heat transfer. Afterward, the warm fluid is pumped towards the TES unit at a constant volumetric flow rate of $F_{W}$, from which the thermal power demand $q_{d e m}$ is covered. Thus, the thermal power recovered during the CHP operation $q_{C H P}$ is stored in the TES, from which a water stream at the temperature of the TES $T_{T E S}$ is transported towards the industrial processes that require it.

Thus, in order to maximize the profit during the operation of a CHP system and to guarantee its proper operation, the costs associated to the resources consumption (e.g., $F_{G}$ and $F_{W}$ ) should be minimized while complying $q_{d e m}$ and, the operating constraints of both the CHP system and TES unit are satisfied. In addition, the costs associated with the systems wear caused by high switching frequency and long operating hours of the CHP system could also be minimized in order to take into account the depreciation of the system.

On the other hand, besides to minimize costs related to the operation of a CHP system, incomes by the sale of the electric power generated could be generated. On the other hand, besides to minimize costs related to the operation of a CHP system, the incomes generated by the sale of the electric power could be maximized as a way to reduce the cost and take advantage of the system outputs. In this regard, in order to improve the profitability of the CHP system, the maximization of revenue could be performed taking into account the energy-price profile and its fluctuations in the market. Thereby, in order to determine the economic-optimal operation of a CHP system along an operation time $T$, the following control objectives are proposed:

\subsection{Costs associated with the resource consumption}

In this case, only the cost associated with the gas consumption is considered since the water flow in industrial environments is generally recirculated through the system as shown in Figure 1 . Thus, the cost by gas consumption $F_{G}$ is defined as follows:

$$
\theta_{1}(k)=\sum_{k=1}^{T} F_{G}(k) P_{r, g}(k),
$$

being $F_{G} \in \mathbb{R}_{\geq 0}$ the volumetric flow of gas consumed by the CHP system, and $P_{r, g} \in \mathbb{R}_{\geq 0}$ the gas price per day in suitable units. It should be noted that, for the case in which the water flow is not recirculated, an equation in the same way of (3) could be defined in terms of $F_{W}$.

\subsection{Costs related to the CHP operation}

Regarding the operation of the CHP system, two types of costs are considered:

- Costs of system depreciation due to the long operation hours of the CHP system, i.e.,

$$
\theta_{2}(k)=\sum_{k=1}^{T} u(k) P_{r, o n},
$$

being $u \in\{0,1\}$ the current state (on/off) of the CHP system, and $P_{r, o n}$ the cost of keeping the system turned on.

- Costs associated with the switching frequency of the CHP system $f_{s w}$, i.e.,

$$
\theta_{3}(k)=\sum_{k=1}^{T} f_{s w}(k) P_{r, s},
$$

being

$$
f_{s w}(k)=|u(k)-u(k-1)| \in\{0,1\}
$$

and $P_{r, s}$ a constant for the cost of switching on/off the system. In should be noted that $\theta_{3}$ is also a way to penalize the high switching frequency of the CHP system to avoid damage system and respect its inertia.

\subsection{Revenues for the sale of the electric power}

For the cases in which the electric power generated $P_{E}$ will be injected to the grid point common for its sale, the total revenues are defined as follows:

$$
\theta_{4}(k)=\sum_{k=1}^{T} P_{E}(k) P_{r, e}(k),
$$

being $P_{E} \in \mathbb{R}$ and $P_{r, e} \in \mathbb{R}_{\geq 0}$ the electric power generated and the sale price of $P_{E}$, respectively. 


\subsection{Trading of electric power}

For the sale of $P_{E}$ to any electric company, some market constraints must be satisfied to avoid economic penalties. In this regard, the amount of electric power traded with the electric companies for a fixed time period must be satisfied with small variations for the whole period. Thus, in order to satisfy the traded electric power, a new control objective that considers the energy market constraints must be included. Assuming that the electric power traded with the electric company $P_{\text {tra }}$ has an updating period $T_{u d}$, for which $P_{t r a}$ must be approximately equal to $P_{E}$, the control objective proposed to minimize differences between $P_{E}$ and $P_{t r a}$ along $T_{u d}$ is defined as follows:

$$
\theta_{5}(k)=P_{r, \Delta P}\left(P_{t r a}\left(k: T_{u d}\right)-\sum_{i=k}^{i=T_{u d}} P_{E}(i)\right),
$$

being $P_{\text {tra }}\left(k: T_{u d}\right)$ the traded power from the time instant $k$ up to the next updating period $T_{u d}, i \in \mathbb{Z}_{\geq 0}, P_{\text {tra }} \in \mathbb{R}_{\geq 0}, T_{u d}<T$, and $P_{r, \Delta P}$ the economic penalty for not fulfilling $P_{t r a}$.

It should be noted that, according to (3) - (7), $F_{G}(k), F_{W}(k)$ and $u(k)$ refer to the inputs of the CHP system, while $P_{E}(k)$ is one of the outputs resulting from feeding such inputs to the system. Thus, according to the previous discussion, in order to determine the optimal operation of a CHP system with an integrated TES (see Figure 1), the economic cost function proposed in this work is defined as follows:

$$
J(k)=-\left(\theta_{4}(k)-\theta_{1}(k)-\theta_{2}(k)-\theta_{3}(k)-\theta_{5}(k)\right),
$$

being $J \in \mathbb{R}$ the total profit for the CHP operation along $T$, which corresponds to the revenues for the sale of $P_{E}\left(\theta_{4}\right)$ minus the total costs $\left(\theta_{1}, \theta_{2}, \theta_{3}, \theta_{5}\right)$ to operate the CHP system.

Thus, in order to achieve the control objective in (8), the activation/deactivation instant of the CHP system and the optimal amount of gas to feed the system should be determined. However, to compute both the revenue and total costs, suitable dynamic expressions are required for both relating the input $\left(F_{W}, T_{1}, F_{G}, u\right)$ and output $\left(P_{E}, T_{2}\right)$ variables of the CHP system, as well as for modeling the heat transfer in the TES unit, i.e.,

$$
\begin{array}{ll}
\psi(k+1) & =f_{1}\left(\psi(k), F_{W}(k), T_{1}(k), F_{G}(k), u(k)\right), \\
P_{E}(k) & =f_{2}(\psi(k)), \\
T_{2}(k) & =f_{3}(\psi(k)),
\end{array}
$$

being $\psi \in \mathbb{R}$ the state vector in the corresponding linear/nonlinear map $f_{1}:\{0,1\} \times \mathbb{R} \mapsto \mathbb{R}$, while $f_{2}, f_{3}: \mathbb{R} \mapsto \mathbb{R}$ are the linear/non-linear maps that relate the states $\psi$ with the output variables for the CHP system.

In addition to the dynamics for the operation of the CHP system, the dynamic for the thermal energy stored in the TES should be modeled in order to guarantee the thermal power demand $q_{d e m}$ and satisfy operating constraints. In this regard, a simplified model based on the energy conservation principle is proposed considering the energy losses by heat exchange with the environment. According to the scheme in Figure 1, at each time that the CHP system is activated, a water stream with flow
$F_{W}$ is pumped from the TES towards the heat recovery unit in the CHP system for its heating. Afterward, this warm flow is returned to the TES where a phenomenon of heat transfer takes place increasing or decreasing the temperature inside of TES $T_{T E S}$ according to the amount of water stored and the others input-outputs flows. One of the output flows of the TES unit is the stream pumped to cover the required $q_{d e m}$. It should be noted that, for this case, the temperature inside the tank is assumed to be uniform, it means the temperature of the output streams will be equal to the temperature inside of TES, i.e., $T_{1}=T_{T E S}$. It worth noting that each flow of hot water pumped from the TES, e.g., towards the CHP system and the building for covering $q_{d e m}$, is returned to the tank after to comply with their functions.

Thus, in terms of heat flow, the dynamic for the energy stored in the TES unit could be defined as

$$
Q_{T E S}(k+1)=Q_{T E S}(k)+\Delta t\left(q_{C H P}(k)-q_{\text {dem }}(k)-q_{\text {loss }}(k)\right),
$$

being

$$
q_{C H P}(k)=F_{W}(k) C_{p}\left(T_{2}(k)-T_{1}(k)\right),
$$

the heat flow per time unit recovered from the CHP system and,

$$
q_{\text {loss }}(k)=U A_{T E S}\left(T_{1}(k)-T_{\text {env }}(k)\right) .
$$

the heat flow per time unit lost by heat transfer with the environment at instant $k$.

In (10)-(12), $q_{\text {dem }} \in \mathbb{R}$ refers to heat flow per time unit required by other processes, $Q_{T E S} \in \mathbb{R}$ is the heat stored in the TES unit, $T_{1}$ and $F_{W}$ are measured inputs, $C_{p}$ is the heat capacity of water, $A_{T E S}$ corresponds to the transfer area of the TES unit, $U$ is the global coefficient of heat transfer, $T_{e n v}$ is the surroundings temperature, and $\Delta t$ refers to the sampling time. Besides, taking into account the physical constraints of the TES unit, it must satisfy $\underline{Q}_{T E S} \leq Q_{T E S} \leq \bar{Q}_{T E S}$ for all $k$, being $\underline{Q}_{T E S}$ and $\bar{Q}_{T E S}$ the lower and upper bounds of $Q_{T E S}$, respectively. Finally, in addition to the process models, other operating constraints such as operating ranges, among others, could be considered in order to guarantee the proper operation of the CHP system.

\section{Proposed Approach}

In order to determine the optimal operation of the CHP system from an economic point of view, the EMPC approach is addressed to design an optimization-based controller that allows maximizing the revenue while minimizing the operating costs along a prediction horizon. From the EMPC approach, the control problem can be transformed into an optimization problem, in which process dynamics, operating limitations, and control objectives will be considered like the constraints and cost function of an optimization problem, respectively. It should be noted that due to the predictive behavior of the EMPC approach, process dynamics are required to perform the prediction of system behavior along a prediction horizon. In this regard, the controller design, the philosophy behind the use of a non-constant 
time step along the prediction horizon, and the model process for the CHP system will be explained below.

\subsection{Controller design}

Based on the receding horizon philosophy [17] and considering a fixed prediction horizon $H_{p}$, the general idea is to predict the input sequences of the system $v(k)=\left[u(k) F_{G}(k)\right]^{T}$ that maximize the profit taking into account both the operating constraints and the time-varying price profiles. Thus, according to the defined control objective in (8), sequences for $J$ and system inputs $v$ along $H_{p}$ can be defined as follow:

$$
\begin{aligned}
\mathbf{J}(k) & \triangleq\left\{J(k \mid k), \ldots J\left(k+H_{p}-1 \mid k\right)\right\}, \\
\mathbf{V}(k) & \triangleq\left\{v(k \mid k), \ldots v\left(k+H_{p}-1 \mid k\right)\right\},
\end{aligned}
$$

with $\mathbf{J} \in \mathbb{R}^{H_{p}}$ and $\mathbf{V} \in\{0,1\}^{H_{p}} \times \mathbb{R}^{H_{p}}$. Thereby, the economic predictive-like controller is based on the following open-loop optimization problem:

$$
\min _{\mathbf{V}(k)} \mathbf{J}(k)
$$

subject to (9) - (10) and

$$
\begin{aligned}
F_{W}(k \mid k) & =F_{w} u(k \mid k), \\
u(k \mid k) & \in\{0,1\}, \\
Q_{T E S}(k \mid k) & \in\left[\underline{Q}_{T E S}, \bar{Q}_{T E S}\right], \\
T_{2}(k \mid k) & \in\left[\underline{T}_{2}, \bar{T}_{2}\right],
\end{aligned}
$$

the logical conditions:

$$
\begin{aligned}
& u(k \mid k)=1 \Longleftrightarrow F_{G}(k \mid k) \in\left[\underline{F}_{G}, \bar{F}_{G}\right], \\
& u(k \mid k)=1 \Longleftrightarrow P_{E}(k \mid k) \in\left[\underline{P}_{E}, \bar{P}_{E}\right], \\
& u(k \mid k)=0 \Longleftrightarrow P_{E}(k \mid k)=0, \\
& u(k \mid k)=0 \Longleftrightarrow F_{G}(k \mid k)=0,
\end{aligned}
$$

and the following safety constraints to avoid the chattering effect and to smooth the behavior of system actuators:

$$
\begin{aligned}
& \Delta u(k \mid k)=0 \Longleftrightarrow 0 \leq \Delta F_{G}(k \mid k) \leq \varepsilon, \\
& \Delta u(k \mid k)=1 \Longleftrightarrow 0 \leq \Delta F_{G}(k \mid k) \leq \mu,
\end{aligned}
$$

being $\Delta u(k \mid k)=|u(k \mid k)-u(k-1 \mid k)|, \Delta F_{G}(k \mid k)=\mid F_{G}(k \mid k)-F_{G}(k-$ $1 \mid k) \mid, F_{w}$ the constant flow of water to recover the heat produced by the CHP system, $P_{E}$ the electric power generated, $\varepsilon$ the maximum variation allowed in $F_{G}$ when the system remains on, $\mu$ the maximum variation allowed in $F_{G}$ when the system switches on, and $\underline{P}_{E}, \bar{P}_{E}, \underline{T}_{2}, \bar{T}_{2}, \underline{F}_{G}, \bar{F}_{G}$, and $\underline{Q}_{T E S}, \bar{Q}_{T E S}$ the lower and upper bounds for $P_{E}, T_{2}, F_{G}$ and $Q_{T E S}$, respectively. Thus, taking into account the nature of variables to be optimized, the optimization problem in (14) is a mixed-integer linear programming (MILP) problem, for which suitable solvers should be

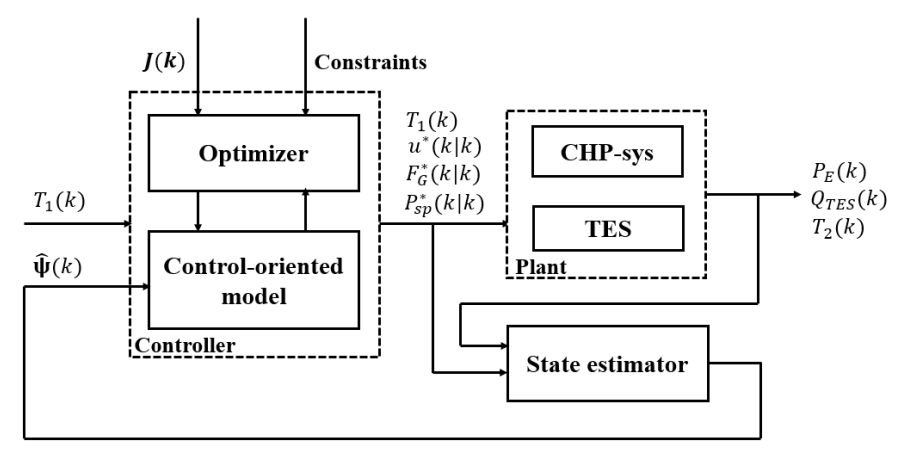

Figure 2: Control scheme for the optimal operation of CHP system with an integrated TES.

chosen in order to solve the problem with a low computational burden.

Assuming that the optimization problem defined in (14) is feasible, i.e., $\mathbf{V}(k) \neq \emptyset$, the optimal sequence $\mathbf{V}^{*}(k)$ exists and, according to receding horizon approach, the first component $v^{*}(k \mid k)=\left[u^{*}(k \mid k) F_{G}{ }^{*}(k \mid k)\right]^{T}$ is sent to the plant. Then, this procedure is repeated for the next instant $k+1$ once measurements of input signals and estimation of the required information about the plant are updated for the next iteration. In order to obtain suitable estimations of model states for the next iterations, an state estimator could be required. The proposed control scheme in real time to determine the optimal inputs along $H_{p}$ is presented in Figure 2.

According to Figure 2, the optimization problem in (14) is solved in real time into the controller module in order to determine the optimal sequence $\mathbf{V}^{*}(k)$. Next, the first components, i.e., $u^{*}(k \mid k), F_{G}{ }^{*}(k \mid k)$, are sent to both the plant and the state estimator. Then, from these signals as well as the real measurements from the CHP system, the current estimation of the model states $\hat{\psi}(k)$ is performed by the estimator and fed back to the controller for the next iteration. Thereby, to estimate the states of the CHP system, a Kalman filter based on the real measurements of the outputs of the CHP system will be designed.

It should be noted that since the electricity trading is usually accounted for and fulfilled every 15 minutes, an unfulfillment of electrical power at the beginning of this time slot/window could be compensated at the end of such time window. Thus, at every time step, the system inputs can be adjusted in order to reduce the difference between the amount of energy traded $P_{t r a}$ and produced $P_{E}$. Taking into account that the way of this restriction is satisfied is one of the contributions of this paper, the trajectory of the prediction model should be updated at time slots lower than a quarter of an hour with the aim to have more degrees of freedom along $T_{u d}$. This last requirement is satisfied using a non-constant time-step size along $H_{p}$ considering time slots lower than 15 minutes during the first part of $H_{p}$, and then increasing the step size to avoid increasing the computational load.

Thus, assume that at each time instant $k$ an optimal sequence is found along $H_{p}$ and the predicted $P_{E}$ from $k$ up to $T_{u d}$ is sold to the electric company. Then, for next iteration $k+1$, the 
Constant time-step size

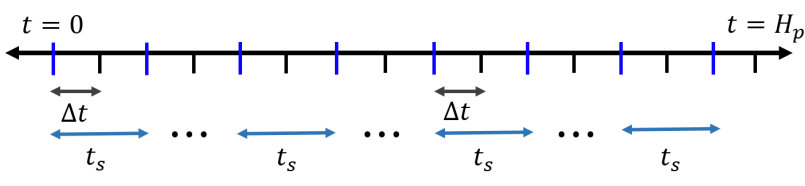

Non-constant time-step size

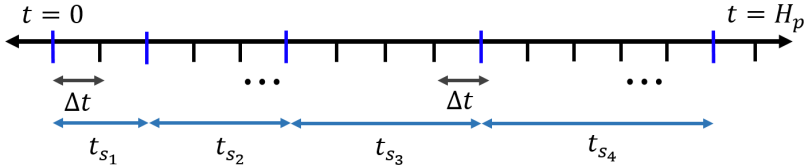

Figure 3: Scheme for the approaches of both constant and non-constant timestep sizes along $H_{p}$.

objective in (8) is minimized in order to guarantee that the sold electric power $P_{t r a}$ can be achieved at instant $T_{u d}$, considering the $P_{E}$ produced from $k$ up to $k+T_{u d}$. Next, when $k=T_{u d}$, a new amount of electric power to be sold is predicted and updated to the electric company, and the process is repeated once again. Thereby, $\theta_{5}$ is considered only along the first 15 minutes of $H_{p}$ since $P_{\text {tra }}$ for this time period has already sold while for the rest of $H_{p}$ new targets can be defined according to the variations of the energy market.

\subsection{Non-constant time-step size}

Due to the high computational cost for solving optimization problems with a large number of variables and the need to solve them fast enough for their implementation in real time, in this paper a non-constant time-step size along $H_{p}$ is proposed. The general idea is to reduce the number of decision variables along $H_{p}$ by using a shorter time-step size at the beginning of $H_{p}$ and increasing the time-step size towards the end of $H_{p}$. That means a greater number of decisions will be considered in the near future while fewer decision variables are considered in the far future. It is worth noting that, the time-step size does not refer to the sampling time $\Delta t$, which is defined by sensing devices and used in the model identification. In contrast, the controller time step, called henceforth $t_{s}$, corresponds to the time instants along $H_{p}$ in which the controller makes decisions, with $t_{s} \geq \Delta t$. A representation of the proposed approach is shown in Figure 3.

Thus, for a given $\Delta t$, which can be established according to the data acquisition devices in the plant or in the modeling procedures, the proposed approach considers using a non-constant $t_{s}$ along $H_{p}$ greater than $\Delta t$. Therefore, according to Figure 3, the controller can only make decisions at the time instants corresponding to the time steps $t_{s_{3}} \gg t_{s_{2}} \gg t_{s_{1}} \geq \Delta t$ and not at each $\Delta t$. It should be noted that, in the strict case $t_{s}>\Delta t$, an internal loop is required, e.g., if $\Delta t=0.1 \mathrm{~s}$ and $t_{s}=1 \mathrm{~s}$, ten iterations for the process model should be implemented. This procedure is commonly known as blocking [17].

Finally, since the optimization problem in (14) explicitly considers models for the generation of both electric and thermal power (9), suitable expressions for characteristic maps $f_{1}, f_{2}$ and $f_{3}$ in (9) are required. Taking into account the complexity of these systems, this paper proposes the identification of datadriven models by using SI methods. The procedure followed to identify the model of the CHP system is explained in the next section.

\subsection{Model of a Combined Heat and Power System}

Although the expressions for the dynamics that take place in the TES unit are given in (10)-(12), suitable expressions for modeling the CHP system will be determined based on real data. In this paper, the SI methods have been selected for model identification as they allow to obtain a state-space representation from input-output data [29, 21, 22]. Thus, a linear approximation for the dynamics of the CHP system (i.e., maps $f_{1}, f_{2}$ and $f_{3}$ ) is considered.

According to Section 2.2, in order to determine the model matrices and the order $N$, this paper focuses on the N4SID algorithm to get proper expressions for maps $f_{1}, f_{2}$ and $f_{3}$ due to its great application and implementation in software $[22,28]$. Therefore, to obtain a suitable state-space representation, proper experiments should be performed for getting information about the real system behavior at different operating conditions. Thus, according to the process shown in Figure 1 , suitable tests were performed feeding different values of $F_{G}$ and $T_{1}$ to the CHP system in order to get its dynamic response and its corresponding outputs. It should be noted that for this case, changes in the water flow were not considered since this flow is considered constant always the CHP system is turned on. However, for those cases in which $F_{W}$ can be modulated, experiments changing this flow could also be required in order to represent the real behavior of the system. Besides, when $F_{W}$ can take different values, it can be added as a decision variable into the optimization problem (14) considering the associated costs.

\section{Case study}

The system to be considered in this paper is based on a real CHP system with an integrated TES unit, which is in the ETA research factory at Technische Universität Darmstadt, Germany. As shown in Figure 1, although the CHP system is formed by different devices, in this paper, the model for each component of the CHP system is not addressed separately. Instead of that, all elements are considered as a joint system, i.e., the CHP. Following this idea, the CHP system will have three inputs, namely, $T_{1}, F_{W}$ and $F_{G}$, which feed the system to produce the outputs $P_{E}$ and $T_{2}$. Although the direct output of the CHP system is $T_{2}$, from this variable, the heat flow recovered $q_{C H P}$ can be computed by using (11).

The CHP system considered has a maximum electric power capacity $P_{E, \max }=6 \mathrm{~kW}$ when the maximum flow of gas $F_{G, \text { max }}=2.4 \mathrm{~m}^{3} / \mathrm{h}$ is fed to the system. Thus, when the system is turned on, the produced $P_{E}$ is sold to the power grid in order to maximize revenue and mitigate the cost associated with the thermal power production $q_{C H P}$, which is the main objective of the CHP system in the ETA research factory. It should be noted that a pump with a constant flow rate of $F_{W}=2753.4 \mathrm{~L} / \mathrm{h}$ is 


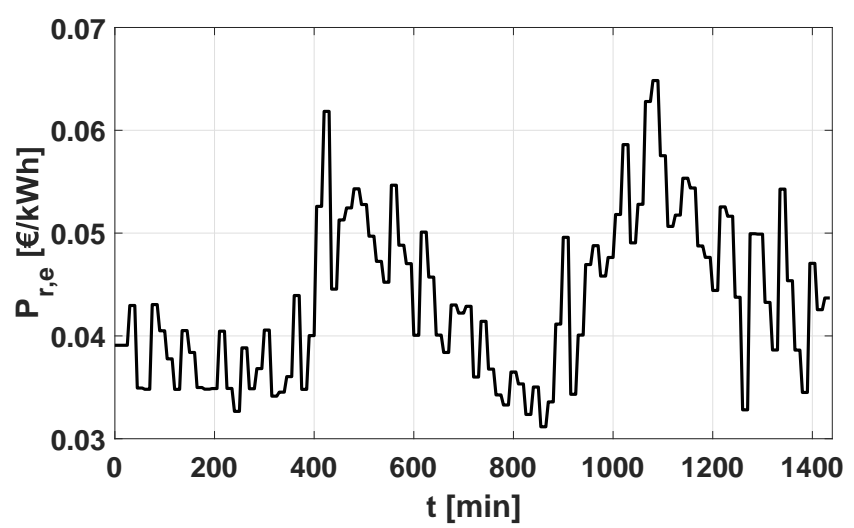

Figure 4: Daily energy price profile for the sale of $P_{E}$.

assumed, and since the fluid is recirculated through the system the associated costs are neglected. The maximum capacity of the TES unit in terms of energy is equal to $\bar{Q}_{T E S}=1000 \mathrm{~kW}$.

On the other hand, in order to get real data, the CHP system is equipped with several sensing devices that provide information in real time about its inputs and outputs. Due to the nature of these systems, their settling times, and the number of variables to be sensed, a sampling time equal $\Delta t=10 \mathrm{~s}$ was chosen to provide data about the real system operation. This information will be useful for both model identification and its validation.

In addition, a prediction horizon of $H_{p}=24$ hours was established to test the proposed approach for one operation day. It means a simulation time of $T_{s}=24$ hours. Thus, taking into account the energy market fluctuations, the daily energy sale price profile presented in Figure 4 was considered into the optimization problem to compute the revenue achieved for the sale of $P_{E}$.

\section{Simulation results}

\subsection{Model identification}

According to Section 4.3, the process model for the analyzed CHP system was identified by using the routine n4sid of the System Identification Toolbox ${ }^{\mathrm{TM}}$ provided by Matlabß. Thus, according to real-data sets from the CHP system in the ETA factory, different values of model order $N$ were tested, and then, the model matrices $A, B, C$, and $D$ were identified with the aim to achieve a high fit degree between the measured and modeled outputs. The model validation for the system outputs is presented in Figure 5.

From the results in Figure 5, it is possible to see that the obtained model properly represents the behavior of both thermal and electric power productions in a suitable way. According to the state-space representation in (2), the identified model matrices are presented in the Appendix.

\subsection{Proposed control scheme}

Based on the case study presented in Section 5 and the proposed control approach in Section 4, a non-constant time-step
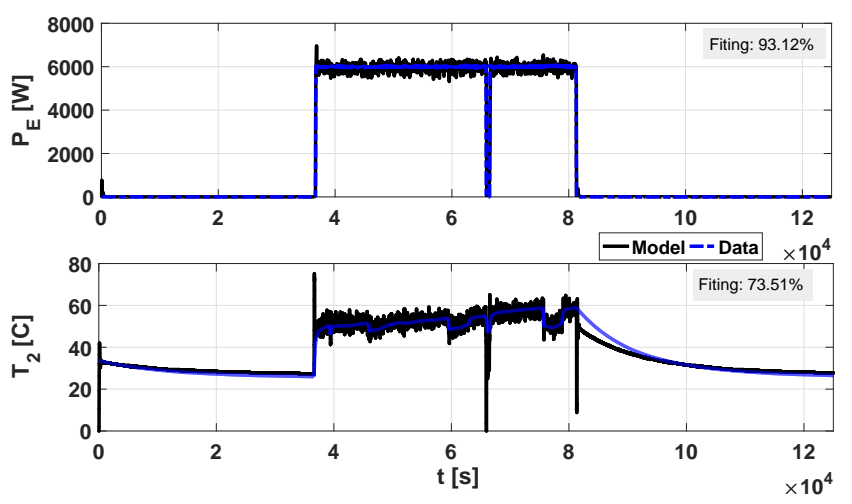

Figure 5: Validation of the CHP model obtained by using SI methods.

size $t_{s}$ along $H_{p}$ is proposed for reducing the computational burden. Thus, given that $\Delta t=10 \mathrm{~s}$ and considering $H_{p}$ as one day, with a traditional predictive control approach a total of 1440 decision variables along $H_{p}$ should be computed for each input variable ( $u$ and $F_{G}$ ). However, the proposed approach states the following decision distribution along $H_{p}$ :

- From $t=0$ to $t=30$ minutes, $t_{s}=5$ minutes and then six elements of the sequences $\mathbf{V}(k)$ are computed.

- Then, from $t=30$ to $t=60$ minutes, $t_{s}=15$ minutes is fixed and two elements of $\mathbf{V}(k)$ are computed.

- After one hour, $t_{s}=1$ hour and the rest 24 elements of $\mathbf{V}(k)$ are computed.

Finally, $|\mathbf{V}(k)|=32$, which corresponds to $H_{p}=24$ hours. On the other hand, since the model for the CHP system runs each ten seconds based on the real data, as explained in Section 4.2 a faster internal loop with an adaptive length that is updated according to the current value of $t_{s}$ was included in the controller design. In this sense, the proposed controller will be executed every five minutes by using a prediction horizon of one day ahead and including the prediction of both the model dynamics and the operation cost according to the current energy market conditions. Then, according to the execution time of the controller, the optimal activation/deactivation sequence of CHP is computed every five minutes taking into account the update of information required by the controller such as the current state of the CHP system and energy market. It means that at every five minutes new optimal scheduling is computed for the next 24 hours.

Thus, given the mixed-integer linear programming nature of the optimization problem in (14), and the need to solve this problem fast enough to react in real time, simulations were developed in Matlabß using the solver IBM ILOG CPLEX Optimization Studio [30] and YALMIP toolbox [31] for stating the optimization problem in the specific format of the solver.

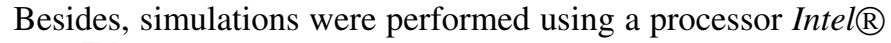
Core $^{\mathrm{TM}}$ i7-5500U CPU 2.40GHz and RAM of 8.0 GB and the parameters in Table 2, in which the costs are presented in economic units (e.u.). 
Table 2: Simulation parameters for the CHP system operation.

\begin{tabular}{cccc}
\hline Parameter & Value & Parameter & Value \\
\hline$P_{r, g}$ & 0.0218 e.u. $/ \mathrm{kWh}$ & $P_{\text {gas }}$ & $11.384 \mathrm{~kW} / \mathrm{m}^{3}$ \\
$P_{r, o n}$ & 5 e.u. $/ \mathrm{kWh}$ & $P_{r, s}$ & 5 e.u. $/ \mathrm{kWh}$ \\
$\bar{Q}_{T E S}$ & $1 \mathrm{MW}$ & $\underline{Q}_{T E S}$ & $2 \mathrm{~kW}$ \\
$C_{p}$ & $4.180 \mathrm{~kJ} / \mathrm{kmol} \mathrm{K}$ & $\bar{T}_{2}$ & $1000 \mathrm{~kg} / \mathrm{m}^{3}$ \\
$\underline{T}_{2}$ & $25{ }^{\circ} \mathrm{C}$ & $\bar{F}_{G}$ & $80{ }^{\circ} \mathrm{C}$ \\
$\underline{F}_{G}$ & $0.5 \mathrm{~m}^{3} / \mathrm{h}$ & $\bar{P}_{E}$ & $2.4 \mathrm{~m}^{3} / \mathrm{h}$ \\
$\underline{P}_{E}$ & $3000 \mathrm{~W}$ & & $6000 \mathrm{~W}$ \\
$\varepsilon$ & $0.3 \mathrm{~m}^{3} / \mathrm{h}$ & $\mu$ & $2.4 \mathrm{~m}^{3} / \mathrm{h}$ \\
\hline
\end{tabular}

The proposed control approach (EMPC) was compared with a typical rule-based control (RBC) implemented in these systems. The underlying idea from RBC is that every time that the system searches one of the operating bounds, the system will be switching on/off depending on the bound achieved. It should be noted that since the RBC does not have predictive behavior, the control objective related to the trading of $P_{E}\left(\theta_{5}\right)$ was not considered when the total operating costs were computed. For the rest of the control objectives, the comparative was performed using the same operating constraints and measurements of the temperature of $T_{1}$.

In Figure 6, the optimal sequences for the activation of the CHP system, and both the water and gas flow fed to the CHP system are presented. From these results, it is possible to observe that RBC has a higher switching frequency than EMPC, which increases the wear of systems and therefore the associated costs. Besides, for each activation of the CHP system, the optimal value found for $F_{G}$ using the EMPC approach presents some small variations that allow modulating the $q_{C H P}$ produced without decrease significantly the generation of $P_{E}$. This last fact could be a consequence of the trade-off between the control objectives of maximizing revenue for the sale of $P_{E}$ and compensate for the total costs. Then, according to the optimal input sequences, in Figure 7, the corresponding outputs of the system are shown. Thus, based on these results, in order to maximize the sale of $P_{E}$, the optimal sequence of $F_{G}$ was set near its upper bound with some small variations to avoid violating the operating range of the TES unit and satisfying $q_{d e m}$.

In addition, from Figure 7 it should be noted that the produced $q_{C H P}$ is always higher than $q_{d e m}$ when the CHP system is turned on. That means the excesses in the thermal power production are stored in the TES for their later use when the system is turned off or even for supplying an unexpected demand. However, for the case in which the EMPC is implemented, the modulation in the values of $F_{G}$ allows that the upper bound of $Q_{T E S}$ will be achieved in greater time than when the RBC is implemented. From this fact, it is possible to keep the system turned on along a long time avoiding turning off the system when reaching its bounds.

On the other hand, in Figure 8, the temperature profiles of the measured $T_{1}$ inside the TES unit, and the obtained $T_{2}$ using both EMPC and RBC are presented. According to the results shown in Figure 8 and the obtained values of $F_{G}$ for each ap-

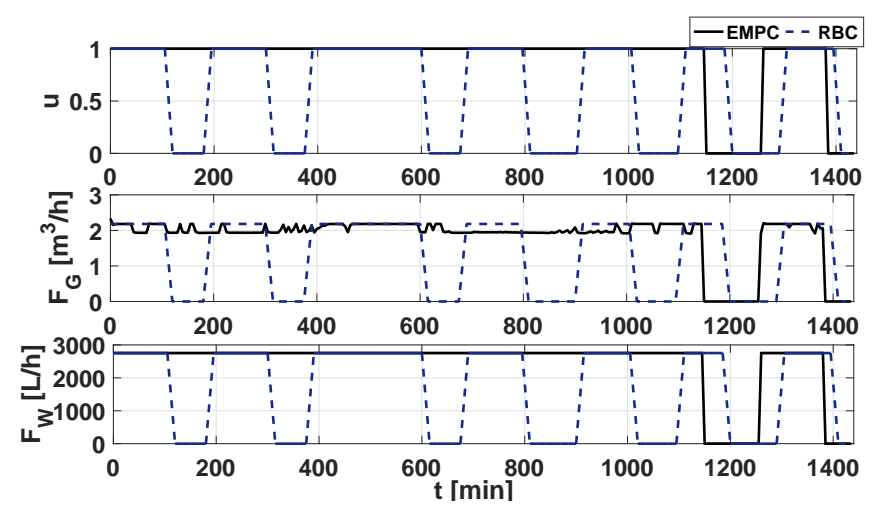

Figure 6: Optimal sequences for the CHP system activation (a), gas flow (b) and water flow (c) for both control strategies.
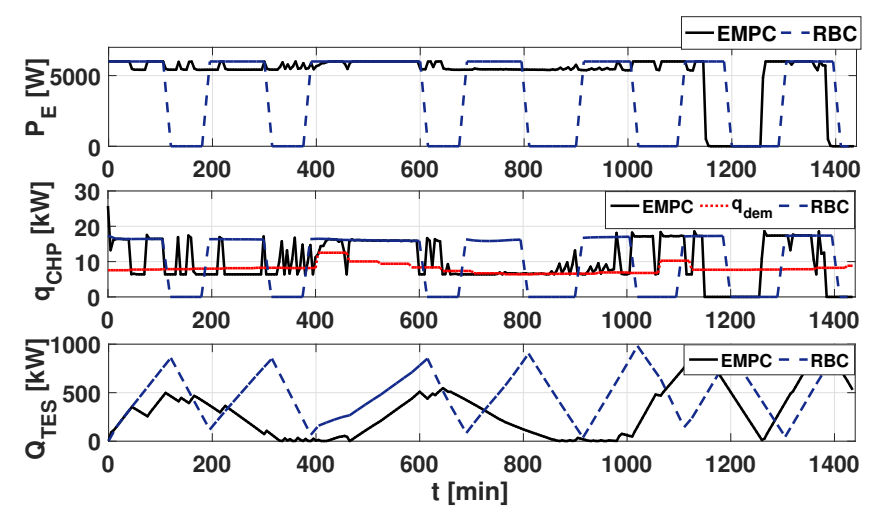

Figure 7: Generated electric power (a), production of thermal power (b), and thermal power level in TES (c) for both control strategies.

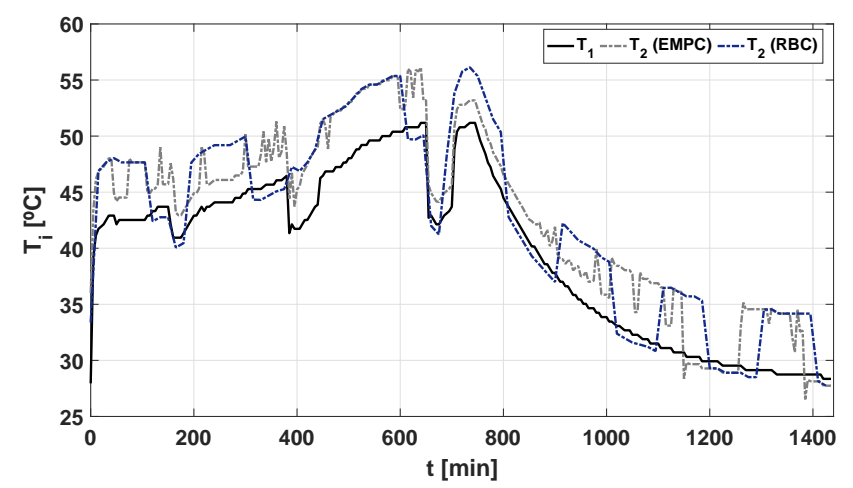

Figure 8: Temperature profiles of both the water inside the TES $\left(T_{1}\right)$ and of $F_{W}$ at the output of the CHP system $\left(T_{2}\right)$.

proach, it is possible to see that higher values of $T_{2}$ are achieved using the RBC. This fact is given since for the RBC approach, the gas flow cannot be modulated and is always pumped at the maximum admissible value.

According to the control objective for the sale of $P_{E}$ in the market and the energy trading constraints, Figure 9 shows a comparison between the generated $P_{E}$ and the traded $P_{t r a}$ at each 15 minutes. From these results, it is possible to conclude 

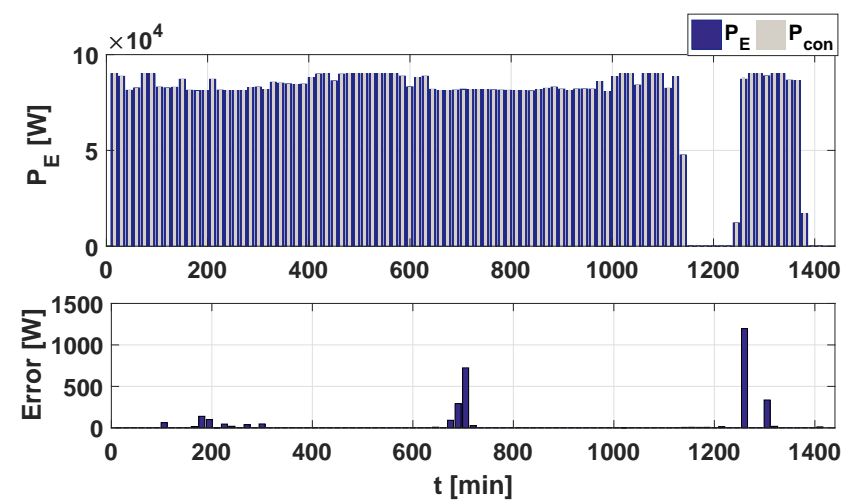

Figure 9: Comparison between $P_{E}$ and $P_{\text {tra }}$ using the proposed approach (a), and its corresponding error (b).

that a high fitting degree between $P_{E}$ and $P_{\text {tra }}$ can be achieved with an error percentage near $0.8 \%$ when the proposed control strategy is implemented. Thus, by using the proposed EMPC approach, it is possible to minimize the differences between $P_{E}$ and $P_{\text {tra }}$ due to the degree of freedom of the controller for adjusting the difference every five minutes before to reach the next $T_{u d}$. It should be noted that results are only shown for EMPC strategy because by using the RBC it is not possible to get a prediction of electric power generation, and therefore, economic penalties cannot be avoided. Thus, including $\theta_{5}$ into the optimization problem, the economic penalties can be reduced or even avoided if a suitable analysis of deviations between these values is performed and reported to the electric company.

Afterward, taking into account the importance to solve the optimization problem fast enough for the implementation in real time of the proposed approach, in Figure 10 the computational time $t_{c}$ spent by iteration along $T_{S}$ is presented. It worth noting that for each iteration, a $H_{p}=24$ hours is considered with the suitable update of price profiles and thermal power demand. Thus, from obtained results, it can be observed that even for a great number of decision variables and long prediction horizons, values of $t_{c}$ lower than five seconds could be achieved when the proposed controller with a non-constant time-step size is implemented. Then, since the time spent on solving the optimization problem is always lower than ten seconds, and according to the controller design the optimal inputs are found and sent to the CHP system at every five minutes, the proposed control strategy is suitable for its implementation in real time. This last fact is a direct consequence of the use of the nonconstant time-step size along the prediction horizon proposed in this paper.

Finally, in Table 3 the operating costs, revenue, and the real cost for one day of operation using both the proposed EMPC and RBC strategies are reported. From these results, it is possible to see that even if the economic penalties by the difference between $P_{E}$ and $P_{t r a}$ are neglected when the RBC is used, cost reductions per day around $11.3 \%$ can be achieved when the proposed EMPC is implemented. Besides, it should be noted that more revenue can be achieved by selling the electric power generated when the proposed controller is implemented since the

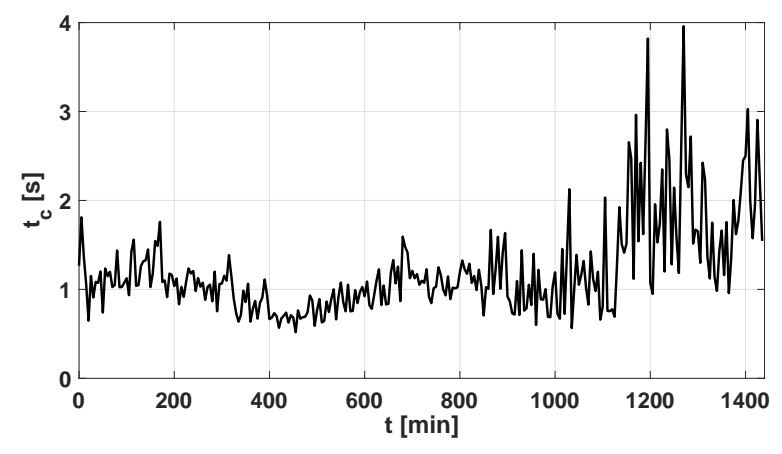

Figure 10: Computational time by iteration along $T_{s}$.

Table 3: Total costs in economic units for one operation day by using both control strategies.

\begin{tabular}{cccc}
\hline Approach & Operating costs & Revenue & Real costs \\
\hline RBC $^{1}$ & 151.73 e.u. & 3.86 e.u. & 147.87 e.u. \\
EMPC & 137.06 e.u. & 5.23 e.u. & 131.80 e.u. \\
\hline
\end{tabular}

fluctuations of energy prices are considered and updated every five minutes into the optimization problem. Thus, according to the price profile, the controller makes the decision to produce more or less $P_{E}$. However, it should be noted that the sale of electric power to the grid point is only a strategy to take advantage of one of the outputs of the CHP system and is not the main objective of this proposal. Therefore, the real costs for the operation of the CHP system in Table 3 were computed as the total operating costs minus the income achieved by the sale of $P_{E}$.

\subsection{Comparative assessment}

In this section, the proposed EMPC controller with nonconstant $t_{s}$ (henceforth associated to EMPC $_{1}$ ) is compared with another EMPC controller but considering a constant $t_{s}$ $\left(\mathrm{EMPC}_{2}\right)$. The underlying ideas behind this comparative assessment are to evaluate the performance of the proposed controller when disturbances take place and to study the lost of optimality due to the reduction in the decision variables in $\mathrm{EMPC}_{1}$ with respect to $\mathrm{EMPC}_{2}$. In order to test both controller designs under the same operating conditions, a new scenario was designed considering disturbances during the CHP operation.

Thus, the general idea is to show the advantages of using a non-constant time-step size $\left(\mathrm{EMPC}_{1}\right)$ defined with shorter timestep sizes at the beginning of $H_{p}$, and, based on the obtained results, to compare the performance of the proposed controller with respect to the conventional $\mathrm{EMPC}_{2}$. This last fact is given since the total number of decision variables along $H_{p}$ is reduced even when more degrees of freedom are considered for the near instants when $\mathrm{EMPC}_{1}$ is used. In this regard, the $\mathrm{EMPC}_{2}$ was designed using a constant time step $t_{s, 2}=30$ minutes along the whole $H_{p}$, i.e., $|\mathbf{V}(k)|=48$. It should be noted that $t_{s, 2}$ has been

\footnotetext{
${ }^{1}$ It should be noted that in this case, economic penalties by not satisfying the trading of electric power are not included.
} 

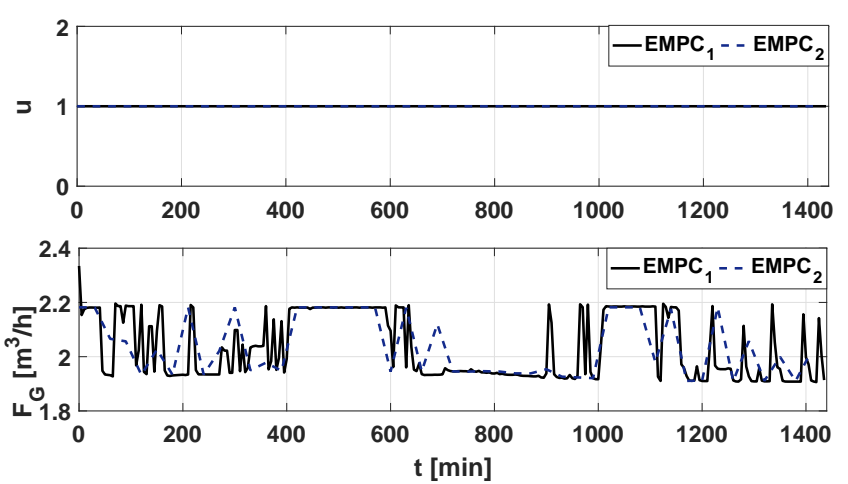

Figure 11: Optimal sequences for the inputs of the CHP system by using $\mathrm{EMPC}_{1}$ and $\mathrm{EMPC}_{2}$.

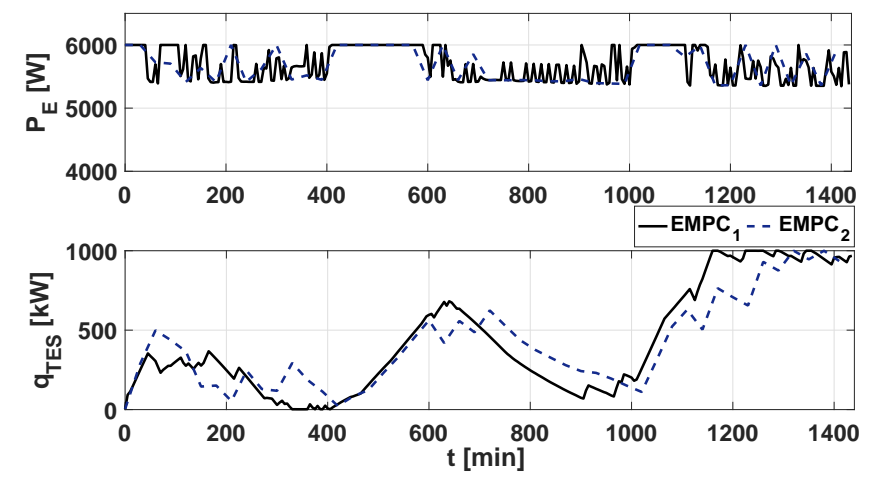

Figure 12: Optimal sequences for the outputs of the CHP system by using $\mathrm{EMPC}_{1}$ and $\mathrm{EMPC}_{2}$.

selected larger than $t_{s, 1}$ since for lower values of $t_{s, 2}$ it was not possible to finish the simulation due to the high computational load.

Two disturbances to simulate a fault in the valve that provides the gas flow $\left(F_{G}\right)$ to the CHP system were simulated. Thus, from $t=60$ minutes up to $t=120$ minutes, a reduction of $5 \%$ in the optimal value of $F_{G}$ fed to the CHP system was considered. Afterwards, in the same way as the first disturbance, from $t=1000$ to $t=1120$ minutes a reduction of $3 \%$ in $F_{G}$ regarding its optimal value was included. The obtained results for both control strategies are shown in Figures 11 and 12. In addition, the error between the traded and produced $P_{E}$ (see control objective $\theta_{5}$ ) for each one of the controllers tested is presented in Figure 13.

Based on the results in Figures 11 and 12, it is possible to observe that even when the proposed EMPC $_{1}$ takes into account fewer decision variables than the $\mathrm{EMPC}_{2}$, both the optimal sequence $\mathbf{V}$ as well as the CHP system outputs have similar behavior in both cases. However, the main difference between both approaches could be better appreciated in Figure 14, where the computational time spent at each iteration is shown for both controllers. According to these results, it is possible to conclude that even when fewer decision variables are considered by using $\mathrm{EMPC}_{1}$, the proposed approach is a suitable strategy

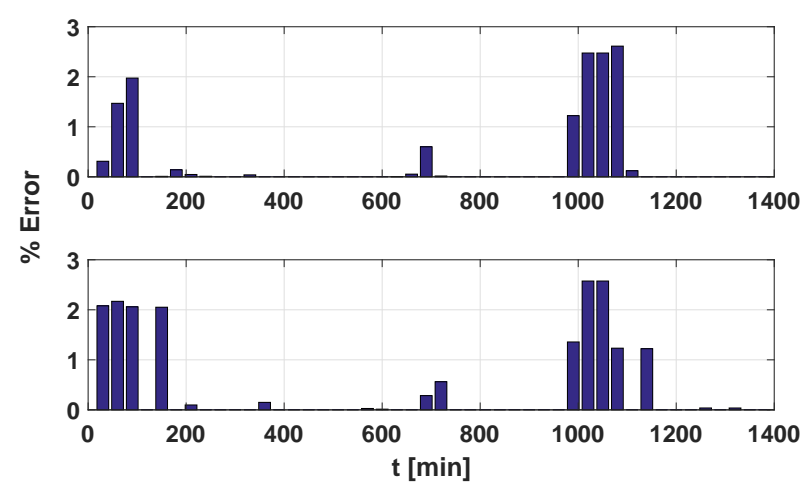

Figure 13: Comparison between $P_{E}$ and $P_{\text {tra }}$ by using EMPC 1 and $\mathrm{EMPC}_{2}$.

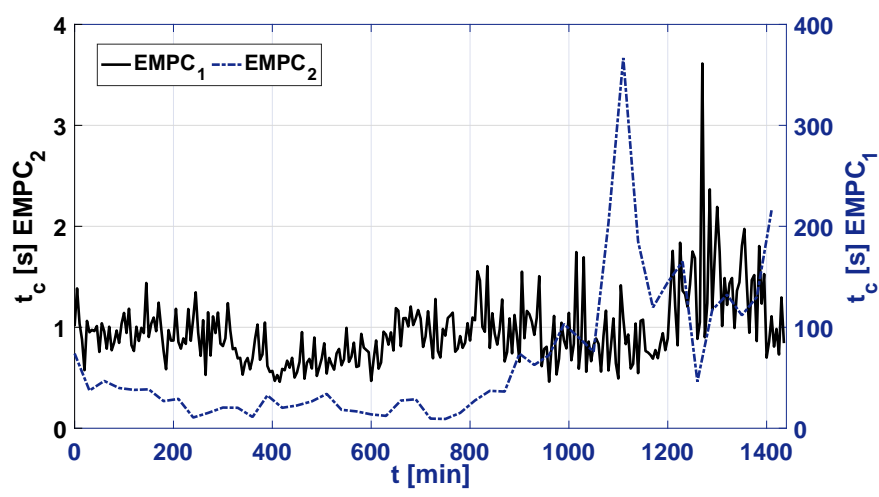

Figure 14: Computational time spent by iteration using $\mathrm{EMPC}_{1}$ and $\mathrm{EMPC}_{2}$.

to reduce the computational load without loss of optimality. Besides, it worth noting that, according to results in Figure 14, the EMPC using a constant time-step size is not suitable for its implementation in real time since the time required to solve the optimization problem is quite large.

It should be noted that, since $\mathrm{EMPC}_{1}$ does a prediction of $P_{\text {tra }}$ for the next 15 minutes and $\mathrm{EMPC}_{2}$ only can do the prediction each 30 minutes due to the value of $t_{s}$, the obtained results using the proposed $\mathrm{EMPC}_{1}$ was organized to compute the differences between $P_{E}$ and $P_{\text {tra }}$ at each 30 minutes. Thus, according to results in Figure 13 and Table 4, differences between $P_{E}$ and $P_{\text {tra }}$ could be reduced up to $2 \%$ for some time intervals ( 30 minutes) if the proposed $\mathrm{EMPC}_{1}$ is implemented. This behavior is given that by using $\mathrm{EMPC}_{1}$ it is possible to define more decision variables in the near future, which brings more flexibility to the controller to be able to achieve the control objectives while satisfying the constraints. Besides, by using shorter $t_{s}$ in the near future along $H_{p}$, the smaller execution time for the controller could be implemented than when $\mathrm{EMPC}_{2}$ is used. From this fact, the real behavior of the CHP system can be updated more frequently and, based on this, either disturbances or non-desired behaviors can be properly managed by the controller. Finally, in Table 4 the operating costs, revenue, and total costs for a simulation day are presented, from which it is possible to see that both strategies achieve similar optimal points. 
Table 4: Total costs for one operation day by using $\mathrm{EMPC}_{1}$ and $\mathrm{EMPC}_{2}$.

\begin{tabular}{cccc}
\hline Approach & Operating costs & Revenue & Real costs \\
\hline EMPC $_{1}$ & 137.12 e.u. & 5.90 e.u. & 131.22 e.u. \\
EMPC $_{2}$ & 132.12 e.u. & 5.87 e.u. & 131.25 e.u. \\
\hline
\end{tabular}

\section{Conclusions}

A predictive-like controller has been designed considering a non-constant time-step size along the prediction horizon and a soft constraint to include the energy-market constraints with the aim to reduce the computational time and minimize the total costs during the operation of the cogeneration plants. In this sense, a lower time-step size has been defined for near instants with the aim to include more decision variables and be able to satisfy the electric power traded and reported to the electric company. Besides, by including energy-market constraints into the optimization problem, the agreements of trading electric power with the electric companies could exist and allow reducing operating costs avoiding the economical penalties and getting revenues for the sale of the electric power.

Based on the obtained results, a lower computational cost for long prediction horizons with a great number of decision variables was achieved without loss of the optimality. In addition, significant cost reductions per day can be achieved while the economic penalties by the differences between the generated electric power and the traded electric power are avoided. In this regard, taking into account that the proposed control strategy is fast enough regarding the energy price updating, a stage of codesign for the implementation of the proposed control strategy in the real system could be developed.

On the other hand, the proposed control strategy could be tested for more complex systems, e.g., a microgrid with two or more different CHP systems and only one TES unit, with the aim to validate its performance. Besides, in order to test the performance of the proposed control strategy and the technique of model identification, scenarios in which disturbances and noise in both the model and measurements take place should be considered.

\section{Acknowledgments}

Authors would like to thank Ikergune (Exte-Tar Group), the project IKERCON (ref. c-10683) and the FI-AGAUR scholarship of the Catalan government for their scientific support in this work. Besides, parts of this work are funded by the German Federal Ministry of Education and Research (BMBF) in the project SynErgie and the German Federal Ministry of Economic Affairs and Energy (BMWi) in the project PHI-Factory.

\section{APPENDIX}

The matrices obtained by using the System Identification Toolbox ${ }^{\mathrm{TM}}$ provided by Matlab $\AA$, and corresponding to the discrete-time identified state-space model, are the following:

$$
A=\left[\begin{array}{cccc}
0.7718 & 0.5131 & -0.2202 & 0.1384 \\
0.1625 & 0.9777 & 0.3117 & 0.2257 \\
0.224 & 0.1613 & 0.691 & -4.852 \times 10^{-4} \\
-0.4279 & -0.4365 & -0.08312 & 0.6417
\end{array}\right]
$$

$$
\begin{aligned}
& B=\left[\begin{array}{ccc}
0.2625 & 0.0002352 & -0.005585 \\
-0.04581 & -9.191 e-05 & -0.003041 \\
0.1334 & 0.0003315 & -0.01392 \\
-0.1721 & -0.0002933 & 0.02044
\end{array}\right], \\
& C=\left[\begin{array}{cccc}
1720 & -8822 & -2238 & -2876 \\
46.48 & -36.18 & -0.08659 & -3.471
\end{array}\right],
\end{aligned}
$$

and $D=0$.

[1] T. Strasser, F. Andrén, J. Kathan, C. Cecati, C. Buccella, P. Siano, P. L. no, G. Zhabelova, V. Vyatkin, P. Vrba, V. Mařík, A review of architectures and concepts for intelligence in future electric energy systems, IEEE Transactions on Industrial Electronics 62 (2015) 2424-2438.

[2] M. Khaljani, R. K. Saray, K. Bahlouli, Comprehensive analysis of energy, exergy and exergo-economic of cogeneration of heat and power in a combined gas turbine and organic rankine cycle, Energy Conversion and Management 97 (2015) 154-165.

[3] G. Zhang, Y. Cao, Y. Cao, D. Li, L. Wang, Optimal energy management for microgrids with combined heat and power (CHP) generation, energy storages, and renewable energy sources, Energies 10 (2017).

[4] A. Costa, A. Fichera, A mixed-integer linear programming (MILP) model for the evaluation of CHP system in the context of hospital structures, Applied Thermal Engineering 71 (2014) 921-929.

[5] B. Aluisio, M. Dicorato, G. Forte, M. Trovato, An optimization procedure for microgrid day-ahead operation in the presence of CHP facilities, Sustainable Energy, Grids and Networks 11 (2017) 34-45.

[6] A. Parisio, C. Wiezorek, T. Kyntaja, J. Elo, K. H. Johansson, An MPCbased energy management system for multiple residential microgrids, in: IEEE International Conference on Automation Science and Engineering (CASE), 2015, pp. 7-14.

[7] W. Gu, Z. Wu, R. Bo, W. Liu, G. Zhou, W. Chen, Z. Wu, Modeling, planning and optimal energy management of combined cooling, heating and power microgrid: A review, International Journal of Electrical Power and Energy Systems 54 (2014) 26 - 37. doi:10.1016/j . i jepes . 2013. 06.028.

[8] Z. Luo, Z. Wu, Z. Li, H. Cai, B. Li, W. Gu, A two-stage optimization and control for CCHP microgrid energy management, Applied Thermal Engineering 125 (2017) 513-522.

[9] C. Marino, M. Marufuzzaman, M. Hu, M. Sarder, Developing a cchpmicrogrid operation decision model under uncertainty, Computers and Industrial Engineering 115 (2018) 354 - 367. doi:10.1016/j . cie. 2017. 11.021.

[10] J. Rawlings, D. Mayne, Model Predictive Control: Theory and Design, Nob Hill Publishing, Madison, WI (USA), 2009.

[11] Y. Zhang, F. Meng, R. Wang, B. Kazemtabrizi, J. Shi, Uncertaintyresistant stochastic mpc approach for optimal operation of chp microgrid, Energy 179 (2019) 1265 - 1278. doi:10.1016/j. energy. 2019. 04.151.

[12] X. Liu, C. J, Economic model predictive control of boiler-turbine system, Journal of Process Control 66 (2018) 59-67.

[13] S. Kuboth, F. Heberle, A. Konig-Haagen, D. Bruggemann, Economic model predictive control of combined thermal and electric residential building energy systems, Applied Energy 240 (2019) 372-385.

[14] H. Tian, Q. Lu, R. Gopaluni, V. Zavala, J. Olson, An economic model predictive control framework for mechanical pulping processes, Control Engineering Practice 85 (2019) 100-109.

[15] M. Ellis, H. Durand, P. Christofides, A tutorial review of economic model predictive control methods, Journal of Process Control 24 (2014) 11561178. Economic nonlinear model predictive control.

[16] J. B. Rawlings, D. Angeli, C. N. Bates, Fundamentals of economic model predictive control, in: 51st IEEE Conference on Decision and Control (CDC), 2012, pp. 3851-3861. 
[17] J. Maciejowski, Predictive control: with constraints, Prentice Hall, Harlow, England, 2002.

[18] J. Wang, J. Wang, P. Zhao, Y. Dai, Proposal and thermodynamic assessment of a new ammonia-water based combined heating and power (CHP) system, Energy Conversion and Management 184 (2019) 277-289.

[19] T. Weber, N. Strobel, T. Kohne, J. Wolber, E. Abele, Realistic modeling of a combined heat and power plant in the context of mixed integer linear programming, Energy Informatics 1 (2018) 27. doi:10.1186/ s42162-018-0037-z.

[20] H. Li, S. Kang, L. Lu, L. Liu, X. Zhang, G. Zhang, Optimal design and analysis of a new CHP-HP integrated system, Energy Conversion and Management 146 (2017) 217-227.

[21] B. Sinquin, M. Verhaegen, A subspace like identification method for large-scale LTI dynamical systems, in: 2017 Signal Processing Symposium (SPSympo), 2017, pp. 1-6.

[22] S. J. Qin, An overview of subspace identification, Computers \& Chemical Engineering 30 (2006) 1502-1513. Papers form Chemical Process Control VII.

[23] J. Diaz C., T. Weber, N. Panten, C. Ocampo-Martinez, E. Abele, Economic model predictive control for optimal operation of combined heat and power systems (2019).

[24] R. Scattolini, Architectures for distributed and hierarchical model predictive control a review, Journal of Process Control 19 (2009) 723-731. doi:10.1016/j.jprocont.2009.02.003.

[25] D. Angeli, A. Casavola, F. Tedesco, Theoretical advances on economic model predictive control with time-varying costs, Annual Reviews in Control 41 (2016) 218-224.

[26] R. Halvgaard, N. K. Poulsen, H. Madsen, J. B. Jorgensen, Economic model predictive control for building climate control in a smart grid, in: IEEE PES Innovative Smart Grid Technologies (ISGT), 2012, pp. 1-6.

[27] P. Overschee, B. De Moor, Subspace Identification for Linear Systems: Theory - Implementation - Applications, Springer US, Boston, MA, 1996.

[28] M. Verhaegen, A. Hansson, N2sid: Nuclear norm subspace identification of innovation models, Automatica 72 (2016) 57-63.

[29] S. Zaiser, M. Buchholz, K. Dietmayer, Rotor temperature modeling of an induction motor using subspace identification, IFAC-PapersOnLine 48 (2015) 847-852. 17th IFAC Symposium on System Identification SYSID.

[30] I. ILOG, Ibm ilog cplex optimization studio, v12. 5, 2013.

[31] J. Löfberg, Yalmip : A toolbox for modeling and optimization in Matlab, in: In Proceedings of the CACSD Conference, Taipei, Taiwan, 2004. 